\title{
On the azimuthal variation of core plasma in the equatorial magnetosphere
}

\author{
D. L. Gallagher and P. D. Craven \\ Space Sciences Laboratory, NASA Marshall Space Flight Center, Huntsville, Alabama \\ R. H. Comfort \\ Center for Space Plasma and Aeronomic Research, University of Alabama in Huntsville \\ T. E. Moore \\ Space Sciences Laboratory, NASA Marshall Space Flighı Center, Huntsville, Alabama
}

\begin{abstract}
Previous results of plasmapause position surveys have been synthesized into a description of the underlying global distribution of plasmasphere-like or core plasma densities unique to a steady state magnetosphere. Under these steady conditions, the boundary between high- and low-density regions is taken to represent the boundary between diurnal near-corotation and large-scale circulation streamlines that traverse the entire magnetosphere. Results indicate a boundary that has a pronounced bulge in the dusk sector that is rotated westward and markedly reduced in size at increased levels of geomagnetic activity (and presumably magnetospheric convection). The derived profile is empirical confirmation of an underlying "tear drop" distribution of core plasma, which is valid only for prolonged steady conditions and is somewhat different from that associated with the simple superposition of sunward flow and corotation, both in its detailed shape and in its varying orientation. Variation away from the tear drop profile suggests that magnetospheric circulation departs from a uniform flow field, having a radial dependence with respect to the Earth that is qualitatively consistent with electrostatic shielding of the convection electric field and which is rotated westward at increased levels of geophysical activity.
\end{abstract}

\section{Introduction}

The Earth is surrounded at low to mid-latitudes $(1<L<\sim 6)$ by a toroid of relatively dense, cold plasma known as the plasmasphere. This region is distinguished from higher $L$ shell regions in that magnetic flux tubes remain exposed to ionospheric filling for extended periods of time. Extended ionospheric filling is primarily the result of plasma circulation on closed streamlines near the Earth. The diurnal motion of the plasma in the plasmasphere is controlled by an electric field induced by the Earth's rotation such that the plasma "corotates" with the Earth. At higher $L$ shells, plasma motion is globally driven by a convection electric field imposed across the magnetosphere by the interaction of the Earth's magnetic field with the solar wind. A stronger convection electric field moves the average boundary between corotating and convective regions to lower $L$ shells, while a weakening of the convection electric field moves this boundary to larger $L$ shells [Nishida, 1966]. Because of the dynamic nature of the solar wind, the convective electric field changes with time, sometimes dramatically.

Plasma flows from the ionosphere into both of these regions of the magnetosphere. Plasma accumulates in those flux tubes corotating with the Earth and can eventually reach a balance,

Copyright 1995 by the American Geophysical Union.

Paper number 95JA02100.

0148-0227/95/95JA-02100\$05.00 corresponding to "filled" magnetic flux tubes, where net outflow is on average quenched. The plasma distribution along corotating flux tubes depends in part upon heating at low and high altitudes and flux tube volume. Plasma densities at higher $L$ shells, the plasma trough, generally remain low due to continuous convection toward the dayside magnetosphere and possible loss at the magnetopause.

The boundary between these plasma regions is often, but not always, characterized by a spacially sharp density gradient called the plasmapause. The plasmapause is variously defined observationally to be the location (in $L$ shell) where the density is between 10 and $100 \mathrm{~cm}^{-3}$. It is also defined to be the location at which the density changes one or more orders of magnitude over a small range of $L$ shell (less than 0.5 ) or azimuth (less than 0.5 hours). In addition, the plasmapause region is associated with the transition between cold, isotropic plasma and field-aligned ionospheric outflows. Small-scale, complex density structure in the vicinity of the plasmapause reflects the dynamic nature of this inner magnetospheric region. Low-density regions are found inside the plasmapause, which may have been emptied due to previous convective forces. High-density regions are found on sunward convection paths, which previously may have been confined and filled within the plasmasphere. The azimuthal stagnation of plasma motion near dusk, due to a near balance between convection and corotation, may also lead to an accumulation of plasma in that region. In addition to the action of convective electric fields, the velocity interchange mechanism of Lemaire and Kowalkowski [1981] may also contribute to 
plasmapause formation. As a result of gravitational and inertial forces, low-density regions spiral toward and high-density regions spiral away from the eventual plasmapause, where these forces are balanced. The dynamic nature of the plasmapause formation, can at times result in multiple gradients that meet the definition of plasmapause reviewed above, that is multiple plasmapause boundaries [Horwitz et al., 1990].

The diumal, or azimuthal, shape of the plasmapause in the Earth's magnetic equatorial plane has been studied for many years. The earliest observers reported both dawn-dusk and noonmidnight asymmetries (see review by Gringauz [1969]). Subsequent observations present a somewhat confused picture, indicating both symmetric and asymmetric azimuthal plasmapause shapes [Chappell et al., 1970; Taylor et al., 1970; Brace and Theis, 1974; Gallagher et al., 1988; Carpenter et al., 1992]. The dawn-dusk asymmetry is characterized by an extension of dense plasma in the evening to greater equatorial distances than at dawn. This bulge in the distribution of dense plasma has been observed to shift sunward and antisunward with increasing and decreasing geomagnetic activity, respectively [Carpenter, 1970; Higel and Wu, 1984; Moldwin et al., 1994].

The observation of an apparent bulge in the evening plasmapause was initially given support by Nishida [1966], where corotational and convection electric fields were superimposed to derive the $\mathbf{E} \times \mathbf{B}$ motions of zero energy ions in the equatorial plane. This simple picture of azimuthal ion motion suggested that the canceling of corotational and convective electric fields at dusk would result in a "tear-drop" like extension of the diurnally closed trajectories of plasmaspheric ions. Subsequent study of the penetration of solar wind impressed electric fields into the magnetosphere and the influence of such effects as finite ionospheric conductivity [Wolf, 1970], dipole tilt [Quegan et al., 1986] (also discussed in the work by Wolf et al., [1986]), and interplanetary magnetic field [Doe et al. , 1992] have lead to a more complex picture of inner magnetospheric plasma distribution with local time.

The steady state azimuthal profile of the plasmapause and its response to changing geophysical conditions remain an observational enigma. Elliptically orbiting spacecraft passing through the plasmasphere reveal a spectrum of observed $L$ shell profiles [e.g., Horwitz et al., 1990], which are separately insufficient to characterize the azimuthal structure. Equatorial, geosynchronous orbiting spacecraft azimuthally sample the radially extended plasmasphere, but it remains difficult to differentiate between azimuthal and radial structures associated with a time-varying plasmasphere [e.g., Moldwin et al., 1994]. Campaigns involving multiple, coincident observations of the plasmapause at varying local times offer the potential of developing snap-shots of radial and azimuthal density structures in the outer plasmasphere, but only for event times and corresponding geophysical conditions. The recent study by Carpenter et al. [1993] does a very good job of discerning plasmaspheric structure in the bulge region for several event times but is unable to fully resolve the spacial and temporal features in the distribution of cold, high density plasma or core plasma. That work differentiates between the main body of the plasmasphere and the bulge region. The plasmasphere is considered to be roughly circular with only a slight bulge at dusk. The bulge is thought to result from the entrainment of plasma removed from the plasmasphere during changing geomagnetic conditions and a possible partial decoupling of high- and lowaltitude convection regimes. The resulting accumulation of core plasma in near evening local times is consistent with the highly structured density distributions observed in this region.
Although determining the transient response of the plasmasphere to changing geophysical conditions continue to require robust studies like that of Carpenter et al. [1993] or the use of innovative remote, global imaging [Williams et al., 1992; Garrido et al., 1994; Frank et al., 1994], the azimuthal profile of core plasma under prolonged steady geophysical conditions can be suggested by combining existing observational studies. The following analysis is based on the results of studies by Higel and Wu [1984], Carpenter and Anderson [1992], and Moldwin et al. [1994]. The works by both Higel and Wu [1984] and Moldwin et al. [1994] are based on observations from geosynchronous orbit $(L=6.6)$. These researchers establish criteria for defining encounters with plasmaspheric plasma and then proceed to statistically quantify the local time width and the centroid of high-density regions as a function of geomagnetic activity. Carpenter and Anderson [1992], using both ISEE I upper hybrid wave and ground whistler measurements, study the plasmapause between 0 and 15 hours magnetic local time, and find it to be statistically symmetric in this local time range. They also quantify the change of the plasmapause location in $L$ shell as a function of geomagnetic activity.

In all three of the referenced studies a systematic, statistical pattern in the location or width of the evening bulge or of the plasmapause location are found. These patterns appear to reveal statistically significant glimpses of an underlying morphology in the distribution of high-density plasma in the inner magnetosphere under steady geomagnetic conditions. When the individually identified patterns are considered collectively, they appear to reveal an equatorial, azimuthal distribution of highdensity plasmas that is otherwise hidden from view. It is this underlying morphology that is the subject of this study, rather than the treatment of those density structures that result from the dynamic processes associated with changing geomagnetic conditions [Chappell et al., 1971; Carpenter and Anderson, 1992; Carpenter et al., 1993; Moldwin et al. 1994].

Our objective is to develop an empirically based description of the azimuthal distribution of core plasma, which we identify with prolonged steady geophysical conditions. We derive a global shape for the distribution of core plasma in the magnetic equatorial plane. The size and orientation of this derived steady state distribution is different for different levels of geomagnetic activity. Plasma densities clearly above normal trough densities are referred to as plasmasphere-like or core plasma. These plasma regions are thought to result from extended exposure to and filling from the ionosphere.

As a result of the potentially distinct origins for evening or bulge plasmas, Carpenter et al. [1993] exclude bulge plasmas from their definition of the "main plasmasphere." A new paradigm of plasmapause formation and thermal plasma transport in the inner magnetosphere is therefore emerging. The origin of bulge plasmas and the processes contributing to plasmapause formation and plasma loss deep within the plasmasphere at substorm onset are being questioned. We suggest that the results presented here describe a basic underlying morphology for the distribution of core plasma and related convection electric fields, that can serve as a reference against the state of the inner magnetosphere under changing conditions.

\section{Observations of the Azimuthal Plasmapause Profile}

Carpenter and Anderson [1991] based their determination of the plasmapause location on measurements from the plasma wave instrument on the ISEE 1 spacecraft. Electron density was determined at magnetic latitudes less than $30 \mathrm{deg}$ and between 0 
and 15 hours magnetic local time. Only those plasmapause profiles for which the number density dropped by a factor of 5 or more over a distance of $\Delta L \leq 0.5$ were used. When multiple plasmapause features were found, only the inner most plasmapause was used. A least squares linear fit was performed between the $L$ value of the plasmapause ( $L_{p p}$ ) and the maximum $K_{p}\left(K_{p \max }\right)$ measured in the preceding 24 hours, resulting in the relation

$$
L_{p p}=5.6-0.46 K_{p \max } .
$$

The preceding one, two, or three values of the 3-hour $K_{p}$ average were ignored for those plasmapause measurements made in magnetic local time intervals centered at 9-, 12-, and 15-hour local times, respectively. No significant variation in $L_{p p}$ was found with local time between 0 and 15 hours MLT.

Higel and $W_{u}$ [1984] used the Relaxation Sounder experiment on the GEOS 2 spacecraft to obtain total density at geosynchronous orbit. Sharp positive and negative density gradients were used to define entry and exit out of the plasmasphere. It was required that the density jump by a relative factor of 2 over three consecutive 12-min spaced samples for a plasmapause to be identified. Density variations from $1 \mathrm{~cm}^{-3}$ per min to several tens of $\mathrm{cm}^{-3}$ per min were observed.

The average MLT between the entry and exit out of the plasmasphere was defined to be the local time centroid $(\Phi)$ of the plasmasphere extension to geosynchronous orbit. A strong correlation was found between $\Phi$ and the average $K_{p}$ for the preceding 9-hours $\left(K_{p 9}\right)$.

$$
\Phi(\text { hours })=23.45-1.92 K_{p 9}
$$

A similar strong linear correlation was found between the local time width $(\Delta \Phi)$ of the plasmasphere at geosynchronous orbit and $K_{p}$. In this case, measurements were restricted to times of relatively steady geomagnetic conditions $\left(K_{p}\right)$ over the preceding 24 hours.

$$
\Delta \Phi(\text { hours })=5.41-1.05 K_{p}
$$

A similar study was performed by Moldwin et al. [1994] using the magnetospheric plasma analyzers on board two geosynchronous satellites, 1989-046 and 1990-095. Measured three-dimensional ion distributions were integrated to obtain number density with a time resolution of $86 \mathrm{~s}$. Average $K_{p}\left(\bar{K}_{p}\right)$ over the preceding 12-hour intervals was related to the local time of the observed plasmaspheric bulge midpoint and bulge local time width. Only those events following steady $K_{p}$ levels were included. A threshold density of $10 \mathrm{~cm}^{-3}$ was used to identify the core plasma. Multiple plasmaspheric encounters were recorded whenever observed densities fell below this threshold for more than $15 \mathrm{~min}$. A linear fit was performed for the bulge local time and for $\bar{K}_{p}>2$.

$$
\Phi(\text { hours })=19.5-0.52 \bar{K}_{p}
$$

At a level of geomagnetic activity of $K_{p}=2$ and below, the core plasma was observed at essentially all local times, therefore no relationship with $K_{p}$ could be established.

The width of the plasmaspheric bulge at geosynchronous orbit was found to take on any value up to a maximum as a function of $\bar{K}_{p}$. That maximum is defined by the expression

$$
\Delta \Phi(\text { hours })=17.2 / \bar{K}_{p}
$$

Moldwin et al. [1994] report that the primary difference between this result and that of Higel and $W u[1984]$ is for $\bar{K}_{p}<2$, although they find bulge widths both more narrow and wider than those found by Higel and $\mathrm{Wu}$ at all levels of geomagnetic activity. The significant difference in sampling rates (one sample every $12 \mathrm{~min}$ versus one every $86 \mathrm{~s}$ ) may have prevented Higel and $\mathrm{Wu}$ from seeing the short timescale structure observed by Moldwin et al. at low $K_{p}$ levels. The longer sampling time of Higel and Wu would result in an effective averaging over the small-scale features observable by Moldwin et al. The consequence will be a smoothing of densities that may lead to measurements that fail to be identified as plasmaspheric encounters by Higel and $\mathrm{Wu}$.

\section{A Composite Picture of the Core Plasma Profile}

If these works can be related, the opportunity exists to obtain a rough picture of the azimuthal distribution of core plasma. Carpenter and Anderson [1992] provide a measure of the radial variation of the plasmapause with changing geophysical conditions. The other papers obtain local time measures of the location and width of core plasma at geosynchronous orbit. Taken together, these works are used here to derive an expression for the steady state azimuthal profile of core plasma, which has implications for global convection electric field models and which may be less sensitive to time-aliased, statistical averages based on single spacecraft observations. Needed assumptions will be stated as we proceed. The implications of those assumptions and the limitations of this approach are discussed in the next section.

We first assume that the radial plasmapause variation defined in (1), for magnetic local times from 0 to 15 hours under changing geophysical conditions (as given by $K_{p}$ ), also defines the proportional change in the radial extent of core plasma at the equator across remaining local times, that is, from 15 to 24 hours. The $L$ shell boundary of core plasma at all local times can then be represented by

$$
L_{p p}=\left(5.6-0.46 K_{p}\right)[1+f(x)]
$$

where the first term is (1) from Carpenter and Anderson [1992] and $f(x)$ is a function that represents the deviation of this plasma distribution from a circular profile as a function of local time. The variable $x$ will be used to measure local time relative to the centroid of the bulge. Although there is evidence that the bulge region may not be symmetric about the centroid [e.g., Carpenter et al., 1992], we will assume symmetry for this exercise. To this point, $f(x)$ is a symmetric function of $x$ but otherwise remains to be defined. Circular, semicircular, and tear-drop profiles for the equatorial distribution of core plasma can all be represented by (6). In the derivation of $f(x)$ that follows, we also assume that the form of this function is independent of the level of geomagnetic activity $\left(K_{p}\right)$. No other assumption for the form of $f(x)$ is made.

Both Higel and $W u$ [1984] and Moldwin et al. [1994] determine a bulge width that decreases with increasing geomagnetic activity. In the case of Moldwin et al., the bulge width is found to vary between small values and the upper bound given by (5). Higel and $\mathrm{Wu}$, however, find a more ordered, less variable, trend given by (3). As discussed above, the differences between these results may be that the maximum bulge width identified by Moldwin et al. includes small-scale plasmaspheric density structures in addition to the general profile. Structures near the plasmapause are frequently seen and may result from surface waves or from continually changing geophysical 
conditions, which lead to the formation of density irregularities both inside and outside the nominal plasmapause location. The much shorter sample time in the Moldwin et al. study may result in their greater ability to see these structures, especially at low levels of geophysical activity when the plasmasphere expands toward $L=6.6$. However, the general trend is the same, that is, the width decreases with increasing $K_{p}$. The objective of this study is to focus on the steady state azimuthal distribution of core plasma, rather than the structures which arise from changing conditions. With this and the longer steady state conditions required by Higel and $\mathrm{Wu}$ for their observations in mind $(24$ hours rather than 12 hours), their description for bulge width will be used here.

The bulge width given in (3) is related to (6) by noting that the bulge boundary will be at $L_{p p}=6.6$ at an azimuth relative to the centroid $(x)$ which is half of the bulge width given in (3) or when

$$
x=\left(5.41-1.05 K_{p}\right) / 2
$$

Letting $L_{p p}=6.6$ in (6) and solving for $f(x)$, we can determine a set of corresponding $f(x)$ and $x$ values as a function of $K_{p}$. With this set of values, a functional relationship can be determined between $f(x)$ and $x$. Such a set of values is plotted in Figure 1 (solid line), along with a least squares fit of an exponential function of a polynomial which is third order in $\mathrm{x}$ (dotted line).

$$
f(x)=e^{\left(-0.03 x^{3}+0.05 x^{2}-0.57 x+0.05\right)}
$$

The values for $f(x)$ and $x$, in Figure 1, are limited to $x$ just under 3 hours, because this corresponds to the maximum bulge width seen by Higel and $W u$ [1984] at geosynchronous orbit. The exponential form of $(8)$ insures that the azimuthal profile reduces smoothly to the constant circular profile of Carpenter and Anderson [1992] for values of $x$ larger than 3 hours. Equation 8 is derived for $K_{p}$ values ranging from 0 to about 5 . Equation 7 for bulge width goes to zero for $K_{p} \approx 5$, consistent with the infrequent detection of core plasma at geosynchronous orbit for very active geophysical conditions. The resulting profiles for $K_{p}$

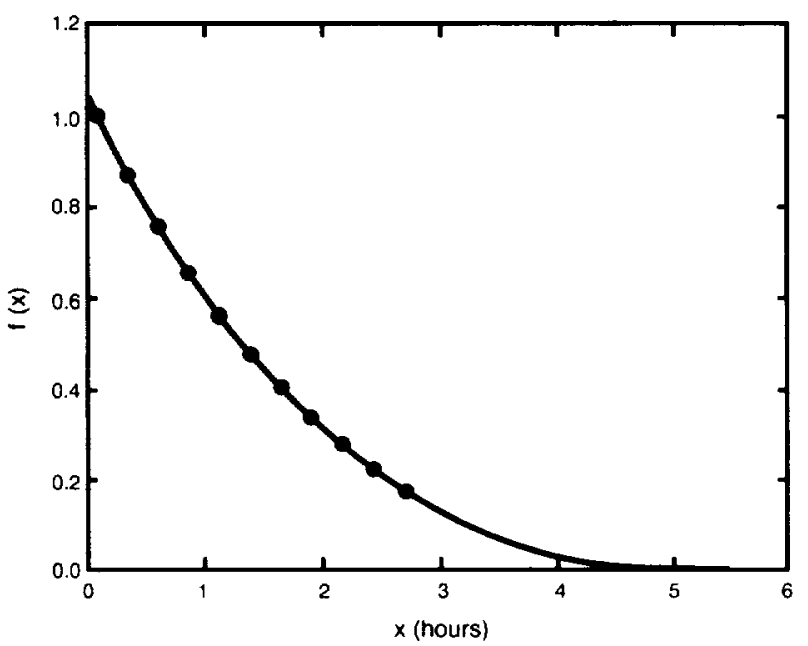

Figure 1. The profile of the bulge $(f(x))$ is derived as a function of the azimuthal rotation from the bulge centroid $(x)$. The function $f(x)$ is based on bulge observations from geosynchronous orbit over a range of geomagnetic conditions (dots are shown every $\Delta K_{p}=0.5$ ). $\approx 1,3,5$, and 7 are shown in Figure 2 as solid lines. Although not unique, these profiles are consistent with the observations of Carpenter and Anderson, Higel and $\mathrm{Wu}$, and Moldwin et al. [1994].

The profiles are shown in Figure 2 relative to the bulge centroid ( $x=0$ hours) and without specific orientation of the bulge relative to the Earth-Sun line. However, both Moldwin et al. [1994] and Higel and $W_{u}$ [1984] report a local time for the bulge centroid which depends on geomagnetic activity. Although Moldwin's observations of the local time of the bulge centroid are scattered for low $K_{p}$, they become less ambiguous for $\bar{K}_{p}>3.5$ and strongly suggest that the bulge does not rotate to a position earlier than about 15 hours MLT. Higel and Wu observations are less ambiguous at lower $K_{p}$ values, perhaps due to their lesser sensitivity to small density features, as mentioned earlier. The larger number of observations in the Moldwin et al. study suggest a more statistically accurate measure of the bulge location for larger $K_{p}$ values, while the greater sensitivity to small scale, possibly transient, features makes the Moldwin et al. study more difficult to use at lower $K_{p}$ values. One approach is to make use of Moldwin et al.'s results at high $K_{p}$ and that of Higel and Wu at low $K_{p}$. Figure 3 shows plots of bulge centroid versus $K_{p}$ from these two studies (solid lines) and a curve fit (dashed line), following this approach,

$$
\Phi=\frac{47}{K_{p}+3.9}+11.3 .
$$

This functional relation approximates the Moldwin et al. result at high $K_{p}$ and the Higel and Wu result at low $K_{p}$. The constants in (9) were derived using a least squares technique but have been rounded and, in the case of the leading constant, slightly reduced to prevent the fitted function from giving a bulge location in local time that is later than the Higel and Wu result at $K_{p}=0$. The application of this result to (6) and (8) is presented in Figure 4. which shows an extended distribution of high density plasma that shrinks and rotates sunward with increasing $K_{p}$.

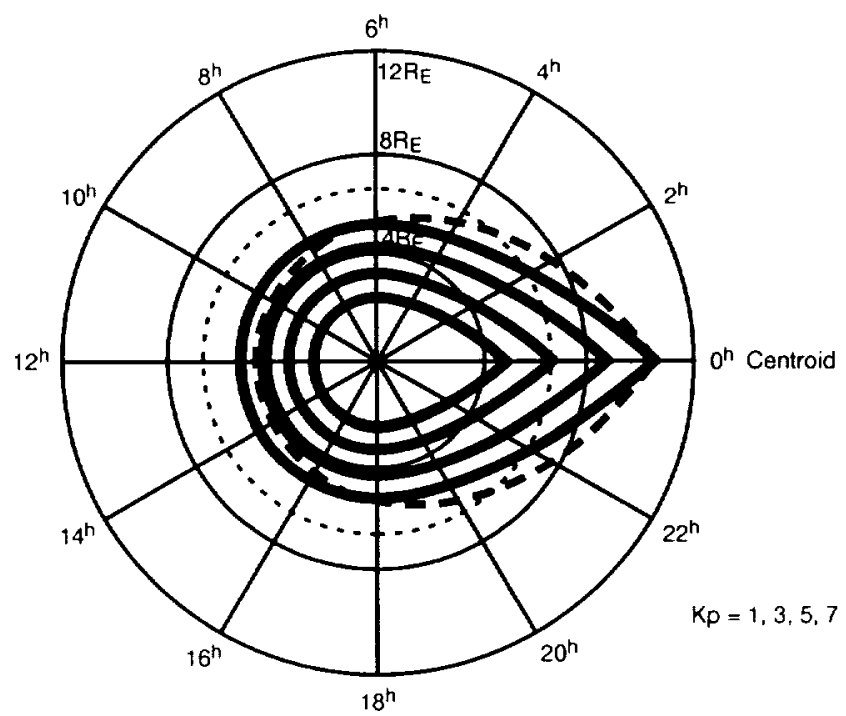

Figure 2. Profiles of core plasma (solid lines) for levels of geomagnetic activity corresponding to $K_{p}=1,3,5$, and 7 are shown relative to the bulge centroid ( $x=0$ hours). The separatrix between convective and corotational flows (dotted line) for a simple, constant cross tail electric field $\left(K_{p}=1\right)$ is also shown. A dotted line marks geosynchrous orbit for reference. 


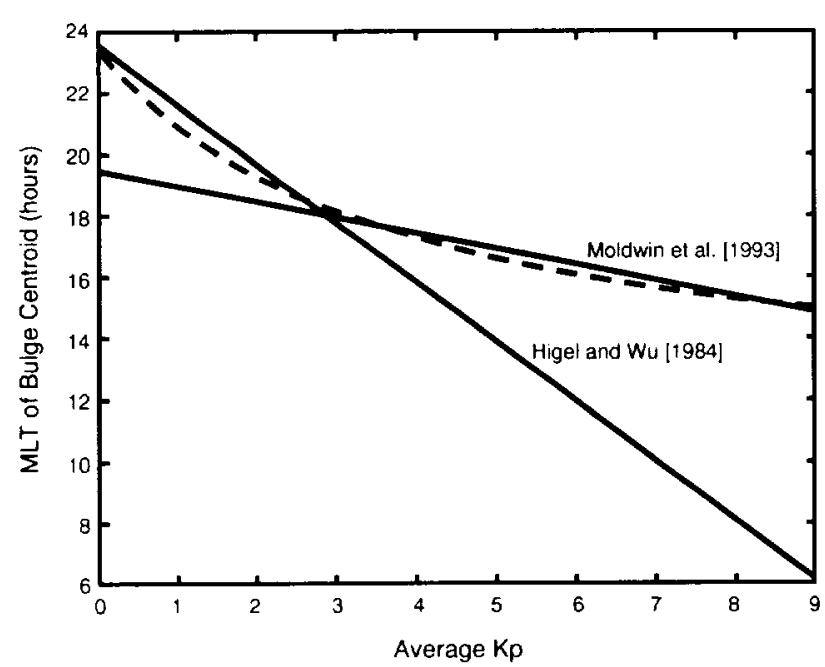

Figure 3. A least squares fit for the magnetic local time location of the bulge centroid (dotted line) as a function of $K_{p}$ is shown along with the bulge centroid determinations of Higel and $W u$ [1984] and Moldwin et al . [1993].

\section{Discussion}

A tear-drop like azimuthal profile for equatorial, core plasma has resulted from combining the observations of Higel and $W u$ [1984], Carpenter and Anderson [1992], and Moldwin et al. [1994]. What does this profile represent? Under what conditions is this picture valid?

One issue is the degree to which the results of these three studies can be directly compared, when Carpenter and Anderson make use of the maximum $K_{p}$ in the preceding 24 hours (with the limitations noted above), while Higel and Wu and Moldwin et al. use the average $K_{p}$ during intervals of steady geophysical activity of 24 and 12 hours, respectively. The maximum $K_{p}$ used by Carpenter and Anderson corresponds to the maximum erosion of the plasmasphere as a result of recent geophysical activity

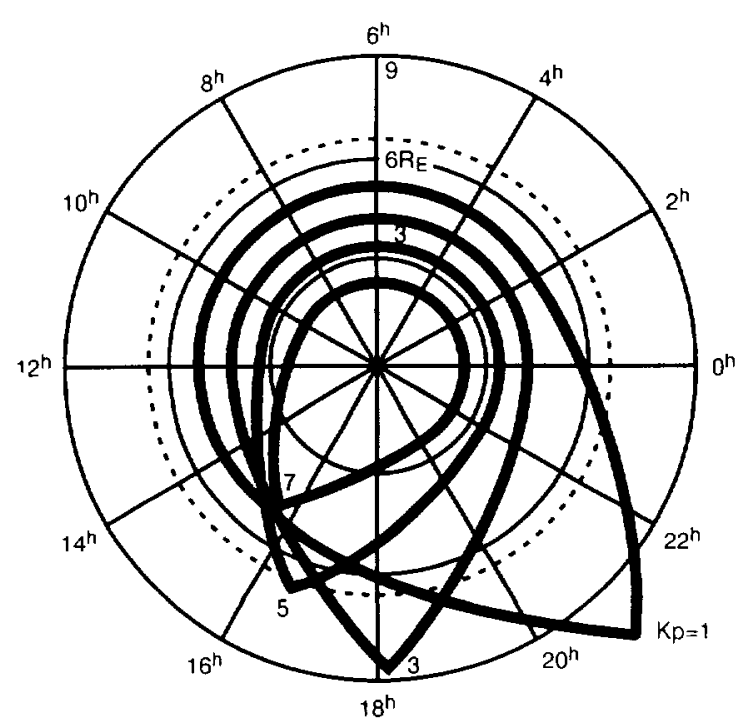

Figure 4. Derived steady state core plasma profiles are shown at four levels of geomagnetic activity corresponding to $K_{p}=1,3,5$, and 7 . A dotted line marks geosynchronous orbit for reference.
[Horwitz et al., 1990], where the erosion process takes place on a timescale of I day or less [Park, 1974]. It can be expected that the innermost plasmapause boundary identified by Carpenter and Anderson would also correspond to the location of the plasmapause, had the level of geophysical activity been held steady indefinitely at the maximum corresponding to each measurement.

The other two studies statistically identify the boundaries of core plasma measured at geosynchronous orbit following $1 / 2$ to I day of steady geophysical conditions. Using whistler observations, Park [1974] determined the time required to refill evacuated flux tubes to saturated density levels to be approximately 1 day for $L=2.5$ and 8 days for $L=4$. Flux tubes exposed to ionospheric filling, however, can be detected on much shorter time scales as filling proceeds (see review by Singh and Horwitz [1992]). The boundaries identified by Higel and $W u$ [1984] and by Moldwin et al. [1994] correspond to regions where core plasma has been entrained by the action of changing convection electric fields or at least where extended ionospheric filling is occurring. In either case, these three studies consistently identify comparable boundaries between plasmasphere-like and trough-like densities at corresponding geophysical conditions, as represented by $K_{p}$.

In part, this determination depends upon the opportunity for a geosynchronous orbiting spacecraft to measure filling flux tubes after 12 to 24 hours of steady geophysical activity. If refilling is characterized by an outward propagating "front" of filled plasma flux tubes, as a result of inner $L$ shells filling quicker than outer $L$ shells [Park, 1974], then many days might be required before significant evidence of refilling becomes available at geosynchronous orbit. If refilling proceeds over a range of $L$ shells, a density shelf might appear [Carpenter and Park, 1973; Horwitz et al., 1984] and be measurable at geosynchronous orbit after only a short period of steady conditions. A simple demonstration of refilling can be made by following the approach of Rasmussen et al. [1993], where diumally averaged filling on a given flux tube is found to approximately follow an exponential asymptotic dependence (see their Figure 4):

$$
n(t)=n_{\mathrm{o}}\left(1-e^{-t / t_{a}}\right)
$$

where $n_{0}$ is the saturation density, $t$ is time, and $t_{a}$ is the time constant for refilling. The time constant across a range of $L$ shells for refilling can be estimated from Park [1974], by assuming that refilling is at least $90 \%$ complete (more than $2 e$-foldings) after 8 days at $L=4$ and linearly scaling to other $L$ shells by flux tube volume. A dipole field is used for simplicity. A level of $90 \%$ is chosen, because a level of $100 \%$ is mathematically impractical with exponential filling and because a $90 \%$ level of filling will be observationally indistinguishable from fully saturated density levels. The density levels associated with saturated and "empty" flux tubes can be taken from Carpenter and Anderson [1991]. At midnight (local time chosen for demonstration purposes only), they find plasmaspheric saturation densities to be given approximately by

$$
n_{e}=10^{(-0.3145 L+3.9043)} .
$$

Minimum density levels in the trough are approximated by

$$
n_{e}=5800 L^{-4.5}+\left(1-e^{\frac{-(L-2)}{10}}\right) \text {. }
$$

The demonstration can be completed by further assuming that the initial $(t=0)$ plasmapause corresponds to that given by 
Carpenter and Anderson [1991] for $K_{p}=5$. This places the plasmapause initially at a low $L$ shell so that filling in outer $L$ shells can be seen. Other than this consideration, there is nothing special about this value of $K_{p}$. The initial $(t=0)$ plasmasphere and trough density profile is shown in Figure 5 as a solid line. The dashed lines show the state of outer $L$ shell refilling after 3 , 6,9 , and 12 hours and after $1,2,3$, and 4 days. It is evident in Figure 5 that the plasmapause defined for $K_{p}=5$ remains observable at least 1 day following the onset of refilling. Refilling proceeds at higher $L$ shells such that a plateau develops like that seen by Corcuff et al. [1972] and Horwitz et al. [1994]. The refilling in Figure 5 is also similar to the theoretical results of Khazanov et al. [1984]. Rasmussen et al. [1993] note that a numerical, first-principles model by Guiter et al. [1991] shows diurnal increases above his simple filling model as flux tubes are carried to the dayside, then later falling back to the simple model at midnight. The densities anticipated at aftemoon and evening local times, therefore, will be somewhat larger than that shown here. A rise in density by a factor of about 4-7 over trough densities is seen after $1 / 2$ to 1 day of filling at geosynchrous orbit, which is consistent with our suggestion that the highdensity regions statistically identified by Higel and $W u$ [1984] and Moldwin et al. [1994] are indicative of flux tubes that will eventually become filled under prolonged steady conditions.

The profiles resulting from the combination of these three studies are show in Figures 2 and 4. Also shown in Figure 2 is a dashed line tracing the separatrix between corotational and convective flows that results from a simple constant cross-tail electric field (for $K_{p}=1$ ) added to the corotational electric field [Roederer, 1970; Lyons and Williams, 1984],

$$
L_{p p}=\sqrt{\frac{c_{1}}{c_{2}}} \frac{\sqrt{1+\sin \Phi}-1}{\sin \Phi}
$$

where $c_{1}=91.5 \mathrm{keV} \cdot \mathrm{R}_{\mathrm{E}}$ and $c_{2}$ has the same units and is chosen such that the stagnation point in the convection model coincides with our bulge centroid. At first glance, the derived distribution of core plasma is remarkably similar to the profile obtained from the simple convection model of (10). Closer examination reveals that these profiles may be observationally distinguishable. The

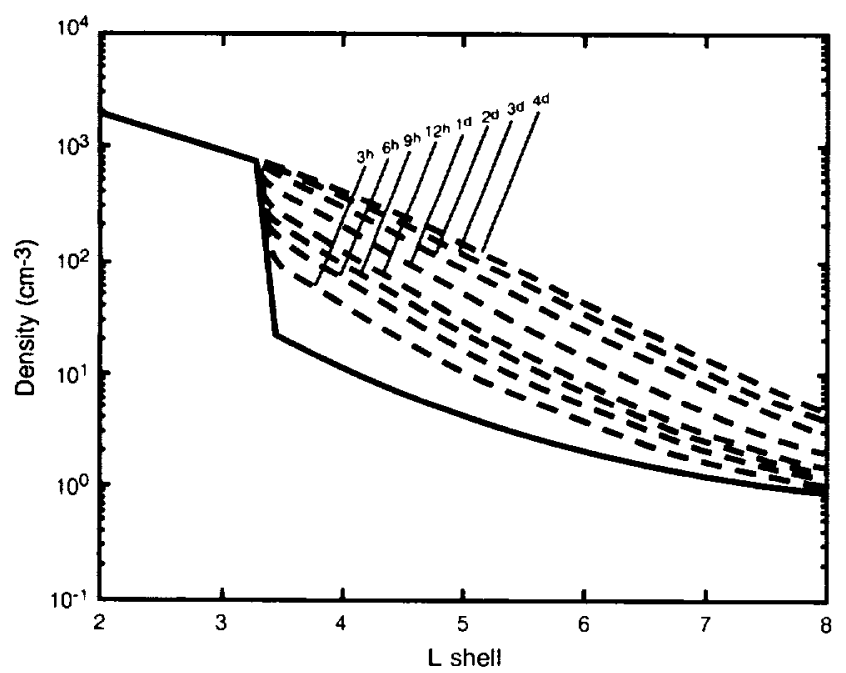

Figure 5. Estimated daily average profiles for refilling at midnight are shown following a maxinum level of geomagnetic activity of $K_{p}=5$ and periods of quiet activity of $3,6,9$, and 12 hours and of $1,2,3$, and 4 days.
Carpenter and Anderson [1992] location for the plasmapause opposite the bulge would need to be in error by nearly $20 \%$ or the Higel and $W u$ [1984] bulge width would need to be in error by nearly $60 \%$ before the profile derived here could be considered consistent with a simple convection model. In Carpenter and Anderson's determination of the plasmapause location (see their Figure 6) there are limited statistics at low $K_{p}$ and significant scatter at higher $K_{p}$ values. Their measurements of the plasmapause position $\left(L_{p p}\right)$ at low $K_{p}$ also suggest possible higher values than that given by the fitted line, rather than a lower value which would be required to match our derived profile with that of the simple convective model.

By changing the value of the parameter $c_{2}$ in $(10)$, it is possible to match the simple convection model to our derived profile on the side opposite the bulge, rather than at the stagnation point. With this change it is found that the simple convection profile does not match the derived bulge width and extension in radial distance. Higel and $W u$ [1984] have limited statistics at low values of $K_{p}$ for determining bulge width, which cannot preclude a wider bulge profile. An error of almost $60 \%$ is required in the Higel and Wu bulge width determination before the derived bulge profile would be consistent with the simple convection model. The Moldwin et al. [1994] study finds bulge widths smaller and larger than those of Higel and $\mathrm{Wu}$, however, it is unable to provide a clear determination of the bulge width due to the wide scatter in observed widths. The maximum bulge widths derived by Moldwin et al. for various values of $K_{p}$ are considerably larger than those found for the simple convection model.

A modification of the simple convection model is made in order to obtain better correspondence between the profiles from simple convection and from this study for $K_{p}=1$ (see Figure 2). At local times opposite the bulge, the influence of the corotational electric field must be extended to somewhat larger $L$ shell, like that which would result from dielectric shielding. At larger $L$ shells an enhancement of the convection electric field over the corotational field may result in a narrowing of convection streamlines at bulge local times. In both $L$ shell

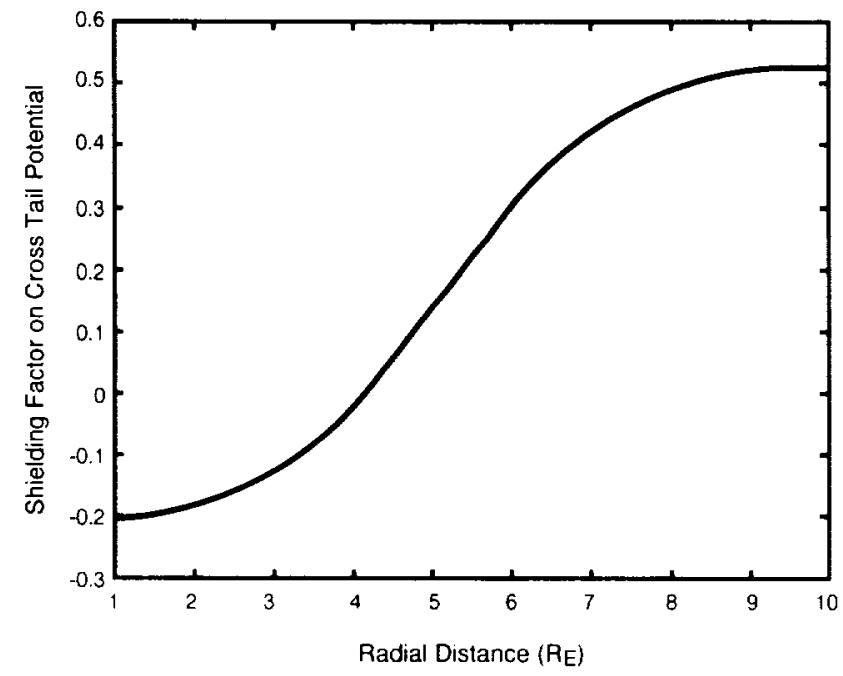

Figure 6. A multipicative factor for the potential corresponding to a constant cross-tail electric field is shown. This factor results from a modification of the simple convection model in order to approximate the empirically derived core plasma profile at $K_{p}=1$. 
regions, a relatively simple modification of the simple convection model is suggested. That modification is accomplished by assuming that a radially dependent multiplicative factor on the electric potential due to simple convection can be derived so as to match the convection separatrix with the derived profile in Figure 2. The total electric potential can then be given as

$$
\Phi=-\frac{c_{1}}{r}+c(r) r \sin \Theta
$$

where the constants $c_{1}$ and $c_{2}$ are from a simple convection model [Roederer, 1970; Lyons and Williams, 1984] and $c_{2}$ has been replaced by $c(r)$. A rough correspondence between this convection electric field model and our derived profile $\left(K_{p}=1\right)$ can be obtained for

$$
c(r)=1.092\left(0.7-e^{-\frac{r^{3.5}}{440}}\right) .
$$

Equation (15) is graphed in Figure 6. What is found is characteristic of a charge separation layer, centered near $L=5$. $L=5$ is also the approximate $L$ shell of the plasmapause away from the bulge for $K_{p}=1$. The implication is that the externally imposed convection electric field results in a global, radially dependent, charge separation that shields the inner magnetosphere. The resulting picture of convection/corotation streamlines, along with our derived profile (heavy line) for $K_{p}=1$, and including our modeled bulge rotation is shown in Figure 7. Similar empirical convection models can be obtained for other levels of geomagnetic activity, but this will be left for a subsequent report. This is not the first time that particle observations have been used to derive a mathematical description of the convection electric field. Mcllwain [1972], for example, used ATS 5 hot plasma observations to derive coefficients for a series of gaussian terms as an approximation of the convection electric potential.

Beginning with a low-latitude barium release in 1964 [Föppl et al., 1965], magnetospheric electric fields have been studied for many years. The influence of the solar wind dynamo on the

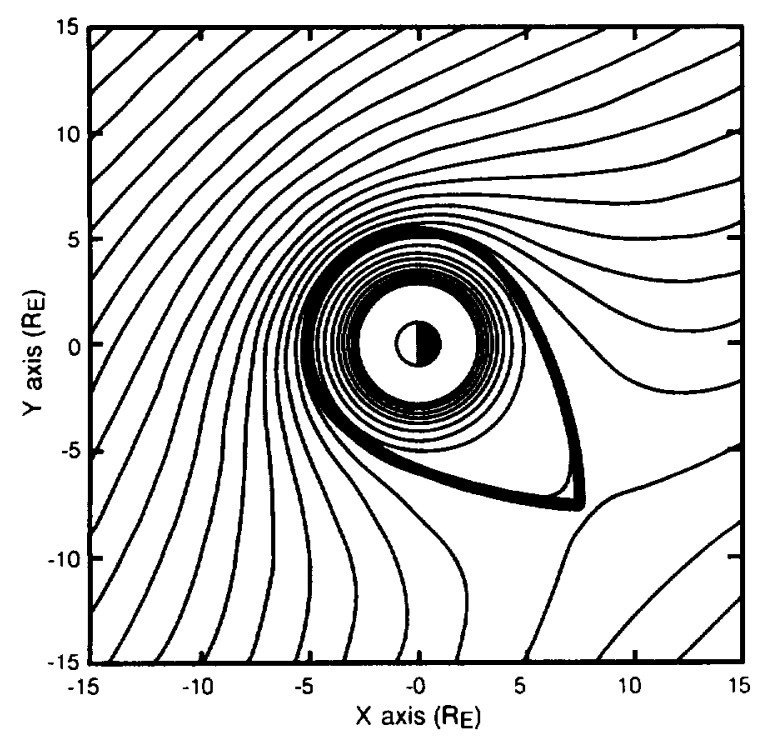

Figure 7. The streamlines for corrotational and convective flows resulting from the modified convection model at $K_{p}=1$ are shown. Also shown (heavy line) is the corresponding empirically derived plasmapause profile. The bulge centroid is rotated to near 21 hours MLT. global distribution of electric fields and associated currents were first studied by Wolf [1970]. Evidence for the shielding of midlatitude to low-latitude from the externally imposed electric field was found in early spaceborne electric probe observations [e.g., Heppner, 1972]. The basic process involves the polarization of the inner edge of the ring current in opposition to the external electric field and the discharge of accumulated polarization charge in the ionosphere. The reader is referred to work such as that by Senior and Blanc [1984] and Siscoe et al. [1991] for further discussion on this topic. Of interest here is that the shielding inferred above as a result of the steady state distribution of dense, cold plasma is at least qualitatively consistent with what is known about ring current shielding of the convection electric field.

Our understanding of the transport of plasma away from the plasmasphere and of the formation of a new plasmapause remains incomplete. The observed disappearance of plasma from the plasmasphere, for example, may occur without requiring plasma to be transported to and lost at the magnetopause. Khazanov et al. [1994] offer an alternative explanation for the observed low densities in the outer magnetosphere. It is shown by Khazanov et al. that flux tubes convected away from the plasmasphere will reach a state of plasma redistribution in the enlarged flux tube on a time scale faster than that required for filling to occur from the ionosphere. The result is a rapid drop in flux tube density as plasma is convected away from the Earth and the apparent evacuation of convecting flux tubes. This analytical work, however, appears inconsistent with observations by Carpenter et al. [1993] of long lasting enhanced density structures outside the plasmasphere. Carpenter et al. [1993] find plasmasphere-like density structures outside the plasmasphere following a substorm and subsequent period of quiet geomagnetic conditions. One possible explanation is that plasma convected away from the plasmasphere and toward the magnetopause is not entirely lost at that boundary to the solar wind. If some magnetic field lines initially convected toward the magnetopause are later turned inside of the magnetopause toward the magnetospheric flanks and convected tailward, continued ionospheric outflow may add to entrained plasmaspheric plasma and lead to enhanced densities over that otherwise observed in the trough. Tailward convection along the magnetospheric flanks from the vicinity of the dayside magnetopause would not result in a continued expansion of flux tube volume and commensurate drop in density, like that which may occur as plasma is originally convected away from the plasmasphere [Khazanov ta al., 1994]. As demonstrated in Figure 5 , significant filling can occur should such flux tubes linger near the Earth for periods of one half day or more.

Moldwin et al. [1994] also see considerable plasmaspheric structure at geosynchronous orbit, especially for quiet geophysical conditions. As discussed earlier, the scatter seen by Moldwin et al. in locating the centroid of the bulge for $K_{p}<2$ relative to the results of Higel and $W u$ [1984] may be due to their greater temporal resolution and selection criteria for identifying plasmasphere-like intervals. The structures seen in the Moldwin et al study at low $K_{p}$ may be related to waves or other density structures often seen in the vicinity of the plasmapause [Carpenter and Anderson, 1991; Carpenter et al., 1992, 1993; McComas et al., 1993]. At $K_{p}=2$ for example, (1) (obtained from Carpenter and Anderson [1991]) gives a plasmapause radius of almost $5 R_{E}$ for local times away from the bulge region. The possibility exists that considerable structure remains in the vicinity of the plasmapause following a return to quiet geophysical conditions. Lemaire and Kowalkowski [1981] report 
that several days may be required for the velocity interchange mechanism to reach a steady state. As a result, the 12 hours of required steady conditions in the Moldwin et al. study may not be sufficient to avoid encountering residual density structures from previous geomagnetic activity.

As stated in the introduction, this analysis stems from statistical evidence for a systematic distribution of core plasma that underlies the widely varying distribution of core plasma typical of changing geomagnetic conditions. The preceding derivation of a shielded, tear-drop convection electric field topology is found to be consistent with this underlying core plasma distribution, but should not be taken as typical of convection electric fields at any given time. Dense regions of plasma detached or extended tail-like from the plasmasphere have been reported by many authors [e.g., Park and Carpenter, 1970; Chappell et al., 1971; Carpenter et al., 1993; Moldwin et al., 1994]. Some features are explained through the use of a time varying convection electric field and the application of shielding [e.g., Grebowsky and Chen, 1976; Spiro et al., 1981]. Many features are not yet understood. As yet unexplained is the persistence of apparently detached regions of dense plasma in the bulge region and well beyond the plasmasphere, even during periods of low geomagnetic activity [Carpenter et al., 1993]. Also not understood are the processes that lead to the formation of sharp plasmapause gradients as a result of increased geomagnetic activity.

The well-known reduction in size of the plasmasphere with increasing convection combines with the rotation of the steady state bulge region detailed in this work to imply a very large change in the plasma environment in the evening local time sector where substorm injection processes are active, as activity increases. As pointed out by Moore et al. [1987], large changes of plasma density also produce large changes in the topography of the index of refraction for magnetohydrodynamic waves, with potential impacts on the nature of transient phenomena with timescales on the order of the wave transit time. In particular, the density gradient of the plasmapause corresponds to an opposite gradient in the fast mode propagation speed.

\section{Conclusions}

Statistical evidence for a systematic underlying structure in the distribution of core plasma in the inner magnetosphere has been synthesized to describe that structure in the magnetic equatorial plane and as a function of magnetic activity. This derived structure was arrived at without advanced prejudice for any specific profile. It is proposed that the statistical evidence and the resulting derivation of a "tear drop" profile for azimuthal core plasma distribution are applicable to conditions of prolonged exposure to constant levels of geomagnetic activity. Conditions that are, however, very unusual in the magnetosphere.

The derived azimuthal profile of core plasma is direct, empirical confirmation of the importance of including the effects of the solar wind induced cross-tail electric field in understanding core plasma convection in the magnetosphere. The differences between our derived underlying distribution of core plasma and the observed distribution during changing geomagnetic conditions is further evidence of the importance of other, still poorly understood, physical processes that influence core plasma motions.

Previously observed westward rotation of the plasmaspheric bulge with increasing geomagnetic activity is confirmed as a feature of the underlying distribution of core plasma. This implies a corresponding rotation of the global magnetospheric circulation flow direction. We are unaware of any theoretical predictions concerning such a rotation, but it seems suggestive of a coherent change in the nature of the magnetospheric wake as convection intensities.

In summary, available studies of the inner magnetosphere from spacecraft operating in different distance ranges from the Earth can be straightforwardly synthesized into a description of the azimuthal distribution of core plasma and the shape of the convection boundary. The shape so derived is reminiscent of that which has been long-associated with the convection boundary in a simple model of sunward flow and corotation, but with significant differences. There is a subtle but observable difference in the detailed shape bearing evidence of some electrostatic shielding, and a more significant gross rotation of the steady state bulge with increasing activity, that must lead to the removal of much of the thermal plasma from the evening region of energetic plasma injections.

Acknowledgments. The authors thank the reviewers for their valuable suggestions in preparing this manuscript. The work of R.H. Comfort was partially supported by NASA grants NAGW-1630 and NAG8-239. Overall, this research was supported by the Office of Space Science and Applications at the National Aeronautics and Space Administration.

The Editor thanks M. B. Moldwin and another referee for their assistance in evaluating this paper.

\section{References}

Brace, L. H., and R. F. Theis, The behavior of the plasmapause at midlatitudes: ISIS I langmuir probe measurements, J. Geophys. Res., 79, 1871. 1974.

Carpenter, D. L., Whistler evidence of the dynamic behavior of the duskside bulge in the plasmasphere, J. Geophys. Res., 75, 3837, 1970.

Carpenter, D. L., and R. R. Anderson, An ISEE/whistler model of equatorial electron density in the magnetosphere, J. Geophys. Res., $97.1097,1992$.

Carpenter, D. L., and C. G. Park, On what ionospheric workers should know about the plasmapause-plasmasphere, Rev. Geophys., $11,133$. 1973.

Carpenter, D. L., A. J. Smith, B. L. Giles, C. R. Chappell, and P.M.E Decreau, A case study of plasma structure in the dusk sector associated with enhanced magnetospheric convection, J. Geophys. Res. , 97. 1157, 1992.

Carpenter, D. L., B. L. Giles, C. R. Chappell, P.M.E. Decreau. R. R. Anderson, A. M. Persoon, A. J. Smith, Y. Corcuff, and P. Canu. Plasmasphere dynamics in the duskside bulge region: A new look at an old topic, J. Geophys. Res., 98, 19,243, 1993.

Chappeil, C. R., K. K. Harris, and G. W. Sharp, The morphology of the bulge region of the plasmasphere, J Geophys. Res.. 75. 3848, 1970.

Chappell, C. R., K. K. Harris, and G. W. Sharp. The dayside of the plasmasphere, J. Geophys. Res., 76, 7632, 1971.

Corcuff, P., J. Corcuff, D. L. Carpenter, C. R. Chappell, J. Vigneron, and $N$. Kleimenova,La plasmasphère en période de recourrement magnétique. Etude combinée des données des satellites OGO 4, OGO 5 et des sifflements reçus au sol, Ann. Geophys. , 28, 679, 1972.

Doe, R. A., M. B. Moldwin, and M. Mendillo, Plasmapause morphology determined from an empirical ionospheric convection model, $J$. Geophys. Res., 97, $1151,1992$.

Föppl, H., G. Haerenel, L. Loidl, R. Lüst, R. Melzner, B. Meyer, H. Neuss, and E. Rieger. Preliminary experiments for the study of the interplanetary medium by the release of metal vapour in the upper atmosphere, Planes. Space Sci., 13, 95, 1965.

Frank, L. A., et al., Imagers for the magnetosphere, aurora. and plasmasphere, Opt. Eng., 33(2), 391, 1994.

Gallagher, D. L., P. D. Craven, and R. H. Comfort, An empirical model of the Earth's plasmasphere, Adv. Space Res., 8, 15, 1988. 
Garrido, D. E., R. W. Smith, D. W. Swift. S. l. Akasofu, R. M. Robinson, and $\mathrm{Y}$. T. Chiu, Imaging the plasmasphere and trough regions in the extreme-ultraviolet region. Opt. Eng., 33(2), 371, 1994.

Grebowsky, J. M., and A. J. Chen, Effects on the plasmasphere of irregular electric fields. Planet. Space. Sci., 24, 689, 1976.

Gringauz, K. I., Low-energy plasma in the Earth's magnetosphere, Rev. Geophys. . 7, 339, 1969.

Guiter, S. M., T. I. Gombosi, and C. E. Rasmussen, Diumal variations on a plasmaspheric flux tube: Light ion flows and $F$ region temperature enhancements, Geophys. Res. Lett., /8. 813, 1991.

Heppner, J. P., Electric fields in the magnetosphere, in Critical Problems of Magnetospheric Physics, edited by E. R. Dyer, pp. 107-120. National Academy of Sciences, Washington, D.C., 1972.

Higel, B., and L. Wu. Electron density and plasmapause characteristics at 6.6 RE: A statistical study of the GEOS 2 relaxation sounder data, $J$. Geophys. Res. . 89, 1583, 1984.

Horwitz, J. L. R. H. Comfort, and C. R. Chappell. Thermal ion composition measurements of the formation of the new outer plasmasphere and double plasmapause during storm recover phase, Geophys. Res. Lett., /l, 701, 1984.

Horwitz, J. L., R. H. Comfort, and C. R. Chappell, A statistical characterization of plasmasphere density structure and boundary locations, J. Geophys. Res., 95, 7937, 1990

Khazanov, G. V., C. E. Rasmussen, Y. V. Konikov, T. I. Gombosi, and A. F. Nagy, Effect of magnetospheric convection on thermal plasma in the inner magnetosphere, J. Geophys. Res., 99, 5923, 1994.

Lemaire $J$., and L. Kowalkowski, The role of plasma interchange motion for the formation of a plasmapause, Planet. Space Sci., 29, 469, 1981.

Lyons, L. R., and D. J. Williams, Quantitative Aspects of Magnetospheric Physics, p. 79, D. Reidel, Norwell, Mass.. 1984.

McComas, D. J., S. J. Bame, B. L. Barraclough, J. R. Donar, R. C. Elphic, J. T. Gosling. M. B. Moldwin, K. R. Moore, and M. F. Thomsen, Magnetospheric plasma analyzer (MPA): Initial three spacecraft observations from geosynchronous orbit, J. Geophys. Res. 98. 13,453, 1993.

McIlwain. C. E., Plasma convection in the vicinity of the geosynchronous orbit, Earth's Magnetospheric Processes, edited by G. M. McCormac, p. 268, D. Reidel, Norwell, Mass., 1972.

Moldwin. M. B., M. F. Thomsen, S. J, Bame, D. J. McComas, and K. R. Moore. The structure and dynamics of the outerplasmasphere: $A$ multiple geosynchronous satellite study, J. Geophys. Res., 99, 11,475, 1994.

Moore, T. E., D. L. Gallagher. J. L. Horwitz, and R. H. Comfort, MHD wave breaking in the outer plasmasphere, Geophys. Res. Lett., 14. 1007, 1987.

Nishida, A., Formation of plasmapause, or magnetospheric plasma knee, by the combined action of magnetospheric convection and plasma escape from the tail, J. Geophys. Res., 7/, 5669, 1966.
Park, C. G., Some features of plasma distribution in the plasmasphere deduced from Antarctic whistlers, J. Geophys. Res., 79, 169, 1974.

Park, C. G., and D. L. Carpenter. Whistler evidence of large-scale electron-density irregularities in the plasmasphere, J. Geophys. Res., $75,3825,1970$.

Quegan, S., G. J. Bailey, R. J. Moffett, and L. C. Wilkinson, Universal time effects on plasma convection in the geomagnetic frame, J. Almos. Terr. Phys., 48, 25, 1986

Rasmussen, C. E., S. M. Guiter, and S. G. Thomas. Two-dimensional model of the plasmasphere: Refilling time constants, Planet. Space Sci., 4I, 35, 1993.

Roederer, J. G., Dynamics of Geomagnetically Trapped Radiation. Springer-Verlag, New York, 1970.

Senior, $C$., and M. Blanc, On the control of magnetospheric convection by the spatial distribution of ionospheric conductivities, I. Geophys. Res., 8c, 261, 1984.

Singh, N., and J. L. Horwitz, Plasmasphere refilling: recent observations and modeling, J. Geophys. Res., 97., 1049, 1992.

Siscoe, G. L., 'N. Lotko, and B.U.Ö. Sonnerup, A high-latitude, lowlatitude boundary layer model of the convection current system, $J$. Geophys. Res., 96, 3487, 1991

Spiro, R. W., M. Harel, R. A. Wolf, and P. H. Reiff, Quantitative simulation of a magnetospheric substorm. 3. plamaspheric electric fields and evolution of the plasmapause, J. Geophys. Res., 86, 2261, 1981.

Taylor, H. A., Jr., H. C. Brinton, and A. R. Deshmukh, Observations of irregular structure in thermal ion distributions in the duskside magnetosphere, J. Geophys. Res., 75, 2481, 1970.

Williams, D. J., E. C. Roeloff, and D. G. Mitchell, Global magnetospheric imaging, Rev. Geophys., 30, 183, 1992.

Wolf, R. A., Effects of ionospheric conductivity on convective flow of plasma in the magnetosphere, J. Geophys. Res., 75. 4677, 1970.

Wolf, R. A., G. A. Mantjoukis, and R. W. Spiro. Theoretical comments on the nature of the plasmapause, Adv. Space Res., 6, 177, 1986.

P. D. Craven, D. L. Gallagher, and T. E. Moore, Space Sciences Laboratory, NASA Marshall Space Flight Center, Huntsville, AL 35812.

R. H. Comfort, Center for Space Plasma and Aeronomic Research, University of Alabama in Huntsville. Huntsville, AL 35807.

(Received August 12, 1994; revised June 2, 1995 ; accepted July 6, 1995.) 
